\title{
Preionization and start-up in the ISX-B tokamak using electron cyclotron heating at $28 \mathrm{GHz}$
}

\author{
A. G. Kulchar \\ The University of Tennessee, Knoxville, Tennessee 37917
}

O. C. Eldridge, A. C. England, C. E. Bush, P. H. Edmonds, G. G. Kelley, C. M. Loring, Y-K. M. Peng, and J. B. Wilgen

Fusion Energy Division, Oak Ridge National Laboratory, Oak Ridge, Tennessee 37830

S. K. Borowski

University of Michigan, Ann Arbor, Michigan 48109, and Fusion Engineering Design Center, Oak Ridge National Laboratory, Oak Ridge, Tennessee 37830

(Received 29 June 1983; accepted 2 April 1984)

\begin{abstract}
A 28-GHz gyrotron is used to produce a plasma at the electron cyclotron resonance in the Impurity Study Experiment (ISX-B) tokamak. The influence of the toroidal magnetic field magnitude, error fields, gas pressure, microwave power, microwave pulse length, and microwave timing is studied for experiments with magnetic field and gas only. Also, experiments with preionization followed by capacitor discharges are carried out in which these quantities are varied, as are the capacitor bank voltages. Optimum conditions of preionization for some of the parameters are determined. A theoretical model that adequately reproduces the data is given. Calculations based on this model show the temporal evolution of the electron temperature and density, the neutral density, and the plasma current. The model adequately accounts for present and previous experimental results and can be used to make predictions for future experiments. The decay of the discharge with no input power is not correctly predicted by the model.
\end{abstract}

\section{INTRODUCTION}

In present designs of tokamak reactors, the inner wall is made thick to protect the circuitry during disruptions and to accommodate a blanket and shield. One purpose of preionization is to start the plasma current with smaller voltages that can diffuse through a thick inner wall. Another purpose of preionization is to reduce the volt-seconds (magnetic flux that are expended in the start-up. This reduction can extend the pulse length of the discharge.

Experiments on plasma production (magnetic field and gas present) and preionization (magnetic field and gas prior to an ohmic discharge) were carried out with electron cyclotron heating $(\mathrm{ECH})$ on the Impurity Study Experiment (ISXB) at Oak Ridge National Laboratory (ORNL). The power for these experiments was produced by a Varian gyrotron operating at a frequency of $28 \mathrm{GHz}$. The power was introduced into the tokamak through a microwave window, and breakdown occurred when the magnetic field was adjusted for electron cyclotron resonance in the ISX-B vacuum system.

This work continued the previous preionization and plasma production study on ISX-B at $35 \mathrm{GHz} .^{1}$ Similar work has also been carried out on a variety of other tokamaks: FT- $1,{ }^{2}$ JIPPT-2, ${ }^{3}$ WT- $1,{ }^{4}$ WT- $2,{ }^{5}$ Tokapol, ${ }^{6}$ and JFT2. ${ }^{7}$ Earlier work was done by Anisomov et al. on a small toroidal device. ${ }^{8}$ Results of theoretical work by Peng et al. ${ }^{9}$ were an important consideration in the planning of these experiments.

Previous experiments in ISX-B showed that preionization caused substantial reductions in the loop voltage during the initial phase of the tokamak start-up. ${ }^{10}$ Transformer flux savings of $30 \%$ in the first few milliseconds were also achieved. The rate of rise of the plasma current was increased by $40 \%$. In the present experiments we adjusted all the available parameters: magnetic field, gas pressure, microwave power, length of microwave pulse, timing of microwave pulse relative to the tokamak firing, and voltage applied to the tokamak primary windings. Optimum conditions for many of these were determined.

Section II contains a description of the experimental arrangement and the available diagnostics. The experimental results are discussed in Sec. III. Section IV contains a theoretical model, a comparison with the data, and scaling to higher frequencies and other tokamaks. The last section contains a summary and conclusions.

\section{EXPERIMENTAL CONDITIONS AND DIAGNOSTICS}

These experiments were done on the ISX-B tokamak, which is described in Refs. 1 and 10. Briefly, the major radius is $93 \mathrm{~cm}$, the minor radius is $27 \mathrm{~cm}$, and the vacuum chamber is rectangular in cross section with a maximum possible elongation of 1.9:1. The magnetic field variation used in these studies was 8.5 to $13.5 \mathrm{kG}$, and the plasma current was typically $100 \mathrm{kA}$. The ohmic heating and vertical field coils are combined into inner and outer poloidal field coil sets, which are separately controlled.

In the normal start-up sequence the toroidal field is energized early and reaches a flat top at -100 to $-10 \mathrm{msec}$. A gas puff prefills the chamber at $-40 \mathrm{msec}$. A capacitor bank is fired to energize the outer winding at $t=0$, and this voltage initiates ionization of the gas. The inner winding is energized by another capacitor bank at a variable time, typically at $t=5-7 \mathrm{msec}$. These capacitor voltages and the timing are preset to center the current in the chamber during the 
start-up phase. The capacitor bank voltages decay at a rate determined by plasma resistance. After about $10 \mathrm{msec}$ the ohmic heating power supplies take over from the capacitor banks and bring the plasma current to a predetermined level. The plasma position and current are sensed, and the feedback control adjusts the inner and outer supplies to maintain the desired position and current.

The microwave power source used was a Varian triplemiter-bend-gyroton with an operating frequency of $28 \mathrm{GHz}$. The power output at the gyrotron window was $100 \mathrm{~kW}$ maximum at the time of the experiment (although the output power measured at the factory was about $160 \mathrm{~kW}$ ). The power was emitted into an oversized waveguide with a $6.35-\mathrm{cm}$ (2.5-in.) inside diameter. Although the tube was designed to operate in the $\mathrm{TE}_{02}$ mode, the triple-miter-bend construction resulted in a mode mixture that was not ideal for longdistance transmission. As a result, the power delivered to the tokamak was not more than $70 \mathrm{~kW}$, as measured calorimetrically with a water load at the end of the waveguide. (During the actual experiments it was impossible to measure the power level.) The power supply was designed to allow pulse widths of $100 \mathrm{msec}$, and these were occasionally used. However, because of the nature of the experiments, pulse widths of $40 \mathrm{msec}$ or less were normally sufficient. The power was produced at times controlled by tokamak timing pulses. For preionization work, the timing was before the actual breakdown produced by capacitor bank firing into the tokamak primary windings. Pulse times as much as $50 \mathrm{msec}$ earlier could be used.

The antenna was mounted on the midplane of the tokamak on the high field side and oriented to launch the power at an angle of $60^{\circ}$ to the magnetic field. The antenna was equipped with a Wengenroth polarizer, ${ }^{11}$ however, with the mode mixture mentioned above, the polarizer was probably ineffective. Hence, it was assumed that it produced power in approximately equal amounts in the ordinary and extraordinary modes.

Standard tokamak diagnostics were used for these experiments. The most important of these for preionization work were the loop voltage $V_{l}$, the plasma current $I_{p}$, visible light from an array of pin diodes, soft $\mathrm{x}$ rays from collimated detectors, current to upper and lower limiters, a double floating probe, and density as measured by a horizontal 2$\mathrm{mm}$ interferometer and a vacuum ultraviolet (VUV) spectrometer. The interferometer measured the line density across the midplane. The VUV spectrometer also viewed the plasma across the midplane. The double floating probe was mounted in the top of the tokamak and could be inserted downward from the wall to the edge of the top limiter at a radial position about $2 \mathrm{~cm}$ outboard of the plasma axis. On one occasion an infrared camera was used to monitor power deposition on the upper limiter.

The limiter current was measured with a commercial current probe on a wire connecting the isolated upper or lower limiter bar to the vacuum tank. These limiters are far from the plasma during normal discharges and only intercept a small plasma current. For the present studies it was observed that current flowed in the curvature drift direction $(\mathbf{B} \times \nabla \mathbf{B})$, as would be expected in a purely toroidal field.

\section{PARAMETER STUDIES}

In an effort to find the best conditions for preionization and plasma production, a series of studies was performed. The studies were motivated by the desire to design a preionization system for a larger tokamak. The optimum pulse length and the optimum magnetic field were determined. In addition, information was gained on the microwave power requirements, the initial pressure, and the optimum timing. Some idea was obtained of how low the capacitor bank voltages could be. Finally, some idea of the effect of field errors was obtained.

\section{A. Optimization of preionization pulse length}

Two of the quantities of interest in scaling studies are the reduction of the loop voltage during breakdown and the concomitant reduction in the volt-second expenditure of the transformer. In previous studies both the dependence of the loop voltage and its time integral on the preionization plasma were studied. ${ }^{I-3}$

The first series of experiments to be described used a microwave pulse of maximum power that was injected some specified number of milliseconds before the capacitor breakdown and overlapped the main discharge by aproximately 1 msec. Figure 1 shows typical oscillograms of the loop voltage with and without a 5-msec pulse of preionization. (The pictures are somewhat different from those reported in Ref. 1 due to a reconfiguration of the capacitors and bus bars since the earlier work.) The large voltage spike noted in Ref. 1 was probably due to a saturable reactor on one of the buses used for earlier work. In Fig. 1, there is a clear difference between the no-preionization and the preionization case, particularly in the first few milliseconds of the discharge. Note the reduction of the measured voltage due to the preionization. In

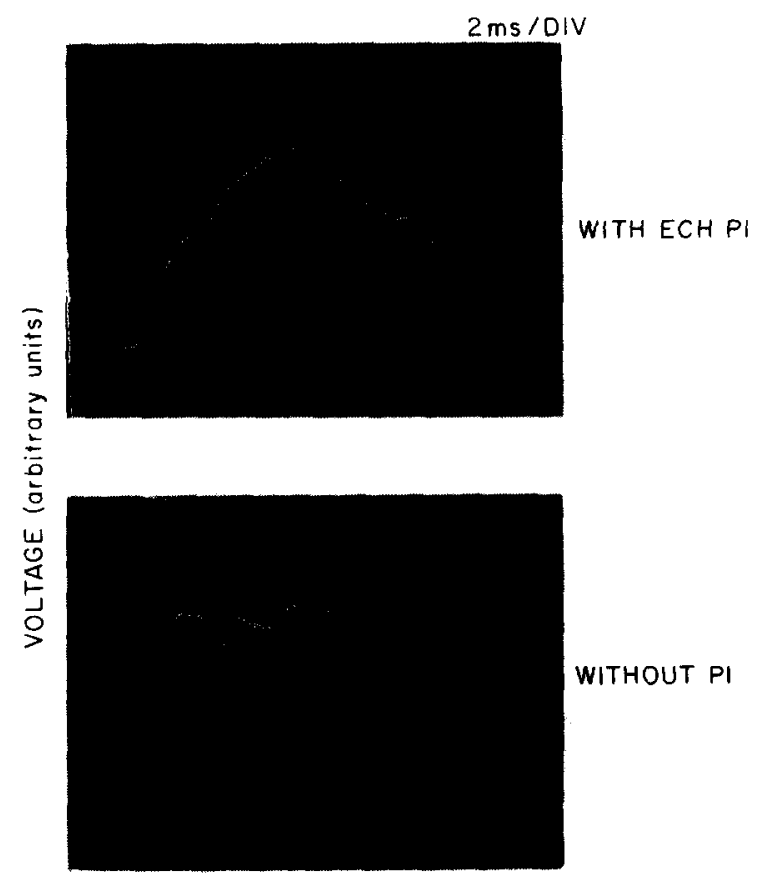

FIG. 1. Measured loop voltage $V_{l}$ as a function of time during the early phase of the breakdown (a) with and (b) without preionization. Note the marked voltage reduction in the first few milliseconds. 
these experiments there is no change in the voltage supplied by the capacitor banks.

In Fig. 2, the reduction in the measured loop voltage at two different times after the start of the tokamak discharge is plotted as a function of the duration of the preionization pulse. In this experiment, the pulse overlapped the main discharge by $1 \mathrm{msec}$ so that slight variations in pulse width would always allow an overlap. Note that the measurement made at the earlier time of $0.6 \mathrm{msec}$ after capacitor bank firing shows a larger effect. However, a $5-\mathrm{msec}$ pulse is sufficient for saturation in both cases. The $2-\mathrm{mm}$ interferometer indicates that the preionization plasma density has reached an equilibrium in 1-3 msec.

A study of the reduction in the loop voltage integral (i.e., the volt-second expenditure) showed that 5 -msec pulses were adequate. An integral over the first $5 \mathrm{msec}$ of the discharge was reduced by $25 \%$ by a preionization pulse of 5 msec, and longer pulses produced no further improvement. Pusles as short as $1 \mathrm{msec}$ produced a noticeable reduction in the integral of $10 \%$.

A series of experiments reconfirmed the finding of Ref. 1 that extending the preionization pulse into the main discharge had no effect. This is probably due to the fact that the microwave power is negligible by comparison with the ohmic power supplied by the capacitor banks during the breakdown phase.

Figure 3 shows the effect of pulse length on the rate of rise of the plasma current. The rate of rise is clearly larger $(50 \%-60 \%)$ with preionization than without it. Again, note that the 5-msec pulse is adequate. No substantial improvement in rate of rise occurs for longer pulses, and there is a large effect for much shorter pulses.

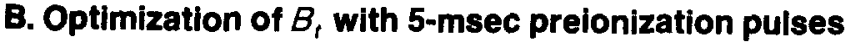

In Fig. 4, the volt-second integral reduction due to the preionization is plotted as a function of magnetic field at the axis of the tokamak $(R=93 \mathrm{~cm})$. In this experiment a $5-\mathrm{msec}$ pulse was used before the main discharge, again with a timing overlap of $1 \mathrm{msec}$. For a field of $10.0 \mathrm{kG}$, the resonance would be exactly at $93 \mathrm{~cm}$. A resonance exists in the vacuum chamber for on-axis fields ranging from $7.1 \mathrm{kG}$ (resonance at the inside limiter) to $13.5 \mathrm{kG}$ (resonance at the outside limiter). The figure shows that the maximum effect occurs at

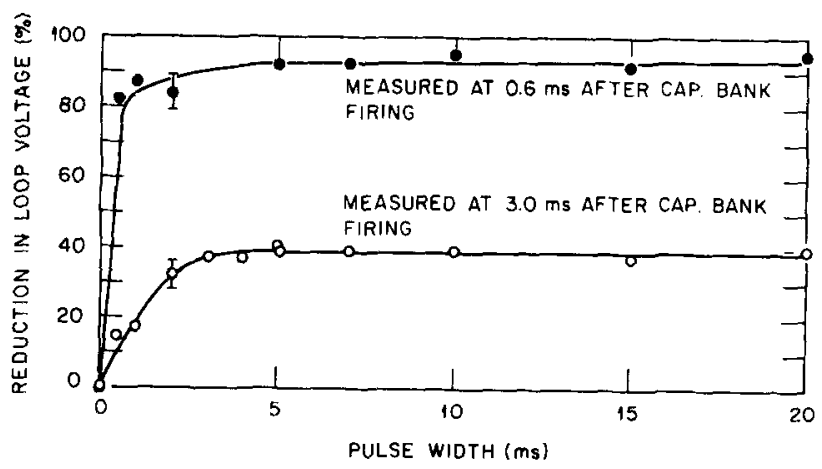

FIG. 2. Reduction in $V_{l}$ at two different times after breakdown as a function of the preionization pulse length. The pulse always overlapped the breakdown by approximately $1 \mathrm{msec}$. The time indicated is the start time before capacitor bank firing. The earlier measurement at $0.6 \mathrm{msec}$ shows a larger effect.

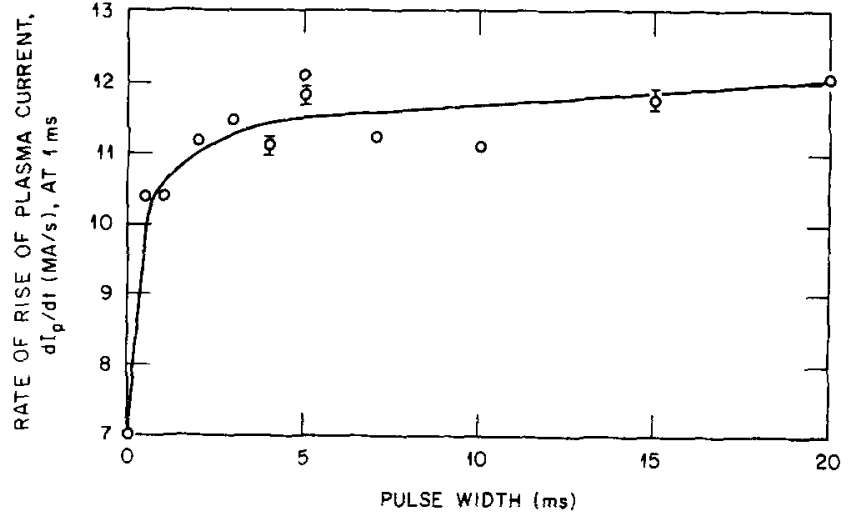

FIG. 3. The effect of pulse length variation on the rate of rise of plasma current $d I_{p} / d t$. The rate of rise is clearly larger with preionization than without it, and a pulse of $5 \mathrm{msec}$ is adequate to achieve saturation.

approximately $11.5 \mathrm{kG}$. At this field, the cyclotron resonance position is displaced toward the outside of the vacuum chamber by $14 \mathrm{~cm}$. For a line density of $2 \times 10^{12} \mathrm{~cm}^{-3}$ and a central field of $11.5 \mathrm{kG}$, the upper hybrid resonance is at the outer limiter $(120 \mathrm{~cm})$. The reduction in the effect for fields higher than $11.5 \mathrm{kG}$ may be due to the absence of an upper hybrid resonance and its concomitant plasma production and heating. Effects were observable for magnetic fields higher than $13.5 \mathrm{kG}$ because there was still some plasma production in the pumping manifold on the outside (low field side) of the tokamak, and the UV light from this aided in the breakdown in the main chamber. On-axis fields below 8.5 $k G$ could not be used since these would have placed the reso-

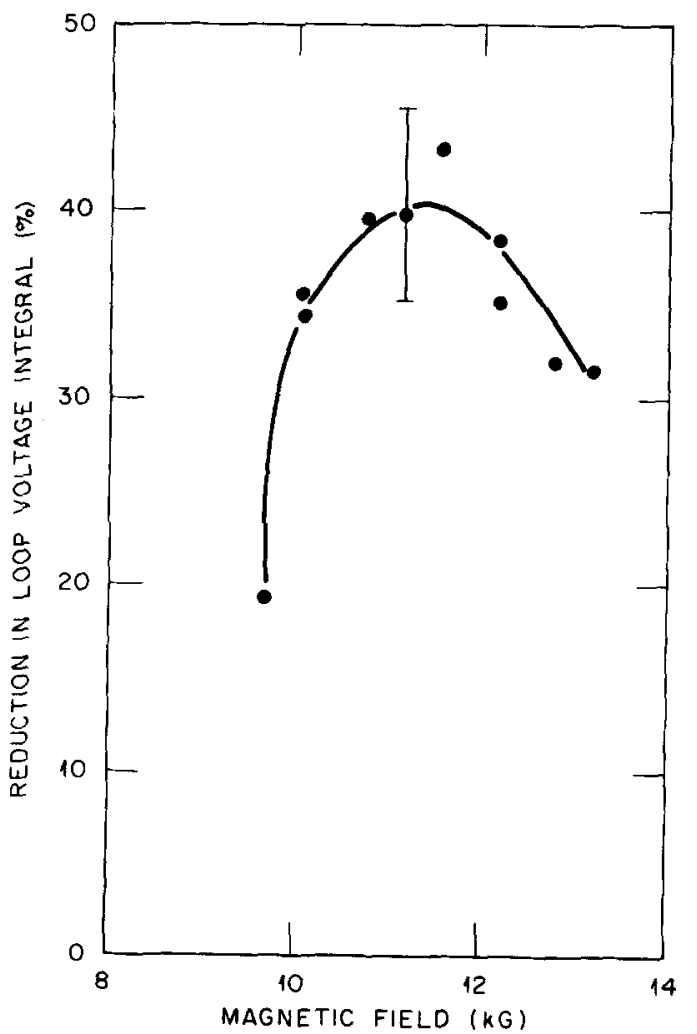

FIG. 4. The reduction in expended volt-seconds for a constant preionization pulse of $5 \mathrm{msec}$ as a function of the toroidal magnetic field on axis. The maximum effect occurs at $B_{t}=11.5 \mathrm{kG}$, which give a cyclotron resonance displaced from the axis outward by $14 \mathrm{~cm}$. 
nance within the waveguide itself, a condition that causes arcing and could easily lead to window failure.

As already mentioned, the plasma current rose faster with preionization than without it, and the current was detectable earlier. In Fig. 5 the rate of rise of the plasma current is shown with and without the $5-\mathrm{msec}$ preionization pulse as a function of the magnetic field on axis. Note that the rate of rise with preionization remains higher at higher magnetic fields than it does without preionization.

The rapid current rise associated with preionization sometimes led to deviations from normal tokamak operation. Ordinarily, based on observation of the in-out position indication, breakdown starts at the outside since the outer windings are energized first. The inner windings are energized a few milliseconds later, stopping the inward motion of the nascent plasma current near the center of the chamber. Eventually the feedback control takes over and centers the current channel. Nonoptimum capacitor bank settings allow the current to start early and give it a more rapid rate of rise. Hence, the current can overshoot the equilibrium position and be lost before the feedback can control it. Adjustment of the capacitor bank voltages and timing can easily prevent this effect.

\section{Variation of microwave power with a 5-msec preionization pulse}

Figure 6(a) illustrates the effect on the loop voltage of varying the microwave power for a $5-\mathrm{msec}$ pulse. Note that there is little effect for a factor of 2 change in power. The gyrotron would not oscillate below a certain minimum beam current, and hence it was not possible to achieve a larger variation in power than this. The effect of varying the if power on the rate of rise of the current for the 5-msec pulse is shown in Fig. 6(b). Again, note the insensitivity to the power. The rate of rise was also measured at $2 \mathrm{msec}$ during the rise with the same result; above some unknown lower power value there is no further effect.

The light amplitude is approximately proportional to the rf power level for the preionization plasma. Also, the value of electron density varies approximately linearly with power. As mentioned earlier, during these experiments the line density was typically $2 \times 10^{12} \mathrm{~cm}^{-3}$ at the normal fill

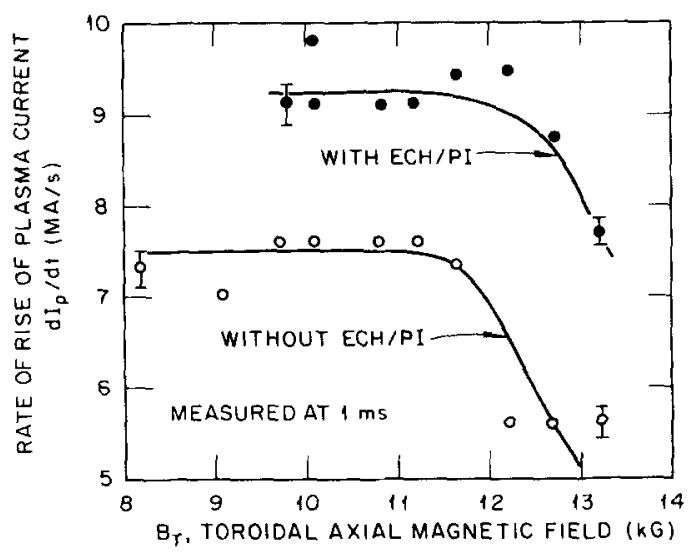

FIG. 5. The effect of magnetic field variation on $d I_{\rho} / d t$ for the preionization experiment of Fig. 4. Also shown is the variation in rate of rise for no preionization. Note the improvement in $d I_{p} / d t$ with preionization.

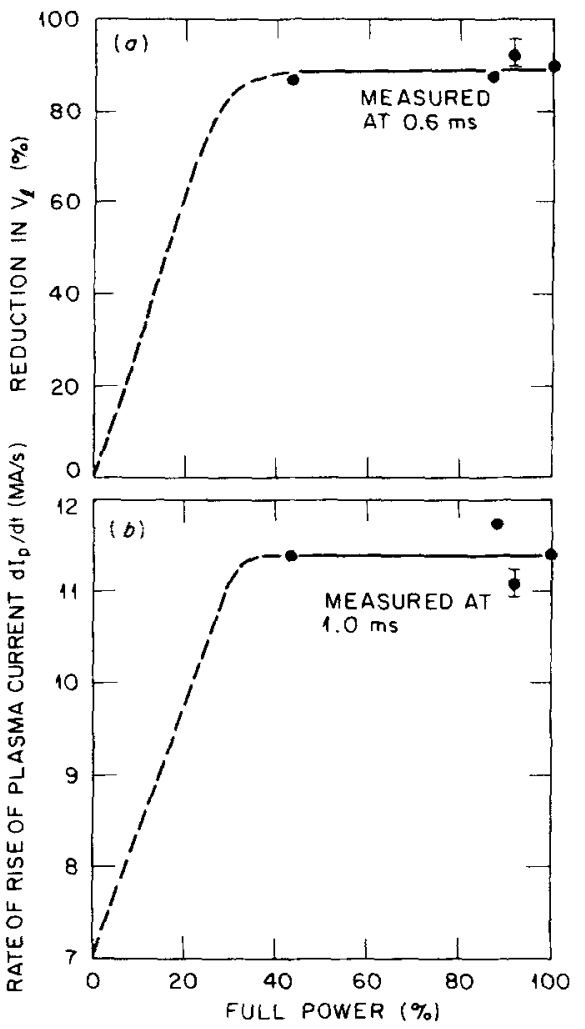

FIG. 6. Effects of $\mathrm{If}$ power variation on (a) $V_{l}$ for a 5 -msec preionization pulse (note the lack of any effect for a factor of 2 variation in of power) and (b) $d I_{p} / d t$ for the same pulse (again, note the lack of effect).

pressure, which is considerably lower than that achievable in the previous work. ${ }^{1}$

\section{Variation of fill pressure with a 5-msec preionization pulse}

The tokamak would not operate over a wide range of fill pressure; hence, the data for this study were limited. Also, only capacitor breakdown plasmas were used to avoid operations problems with the main power supplies. A prefill pulse of gas normally filled the chamber with gas $40 \mathrm{msec}$ before breakdown. Variation of the initial gas input by a factor of 4 caused no change to the loop voltage with preionization. Similarly, there was no change in the transformer volt-second expenditure. The current rate of rise was also independent of the prefill gas puff input for a constant preionization pulse. The interferometer showed a rise in density proportional to the gas puff input and the light from the pin diodes increased proportionally to it. However, the limiter current dropped as the fill pressure was raised.

\section{E. Variation of timing of preionization pulse}

Several studies were made of the effects of timing of various lengths of preionization pulses. These were all done at full power. Figure 7 shows the volt-second saving as a function of the timing of a constant $20-\mathrm{msec}$ preionization pulse. Note that there is still an effect on the tokamak discharge for a pulse that ended $30 \mathrm{msec}$ before the capacitor firing. This is evidence of a long-lived plasma component in the purely toroidal field of the tokamak (i.e., without a rota- 


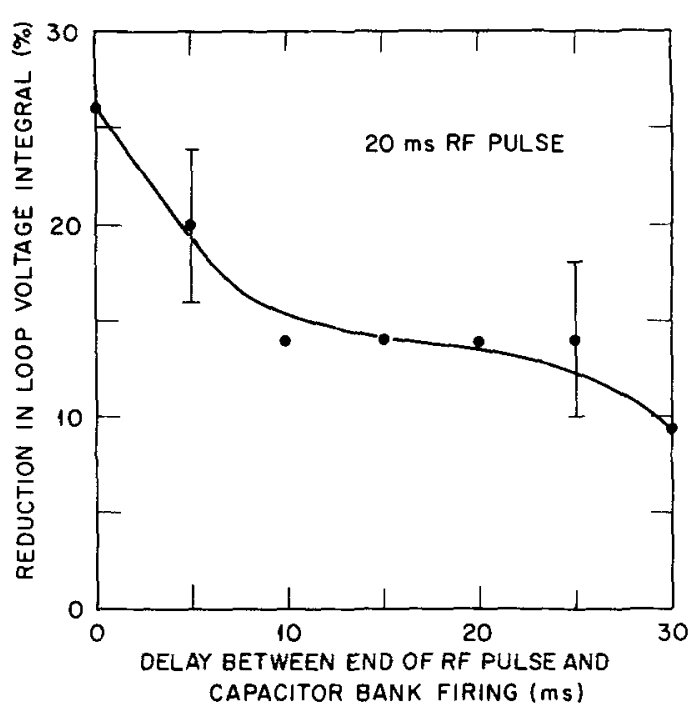

FIG. 7. Percentage saving of expended volt-seconds versus timing of a 20 msec rf pulse. The saving is plotted against the time between the end of the preionization pulse and the capacitor bank firing. The persistence of the effect for a 30-msec delay implies a long-lived plasma component.

tional transform from a plasma current). As the pulse was moved toward the time of the capacitor firing, there was an increase in the volt-second saving until the pulse overlapped the breakdown. Measurements of plasma density by the 2 $\mathrm{mm}$ interferometer showed a density of $\sim 2 \times 10^{12} \mathrm{~cm}^{-3}$ during this pulse, with an $e$-folding decay of about $10 \mathrm{msec}$.

The light signals showed a long decay but with additional features. Figure 8 shows several of the diagnostic signals as a function of time for an early $20-\mathrm{msec}$ preionization pulse that ended $25 \mathrm{msec}$ before the ohmic heating start-up: the limiter current, the radiometer signal, the interferometer, and the pin diode light, with the microwave pulse signal as a timing mark. The light signal always starts with a bright spike that quickly decays and then rises slowly during the pulse. The spike is due to the opening of the gas valve at $-40 \mathrm{msec}$. However, after microwave turnoff the light begins to decay and then displays two peaks before finally disappearing. The density signal shows steps due to the automatic fringe counter; this limits the resolution but the density clearly shows a long decay. The limiter current shows a slow 10-msec buildup and a 2 -msec decay. The radiometer signal also shows an increase during preionization, but some of this is direct pickup.

There are several possible explanations for the long containment time observed. The light buildup and decay could indicate the presence of superthermal electrons. If the electrons are accelerated by the microwave power to energies well above the peak in the ionization cross section, then after microwave turnoff these electrons might decay in energy until they begin to ionize the gas more efficiently. The visible light peaks could be due to the electrons decaying in energy through the region where the gas is easily ionized. The presence of a vertical error field can offset the curvature drift for a class of electrons with a given perpendicular energy and thus permit their containment. ${ }^{12,13}$ These fast electrons would constitute a small toroidal current. No such current was observed within the accuracy of the measurement, which was $1 \mathrm{kA}$. For ISX-B, electrons of approximately 20
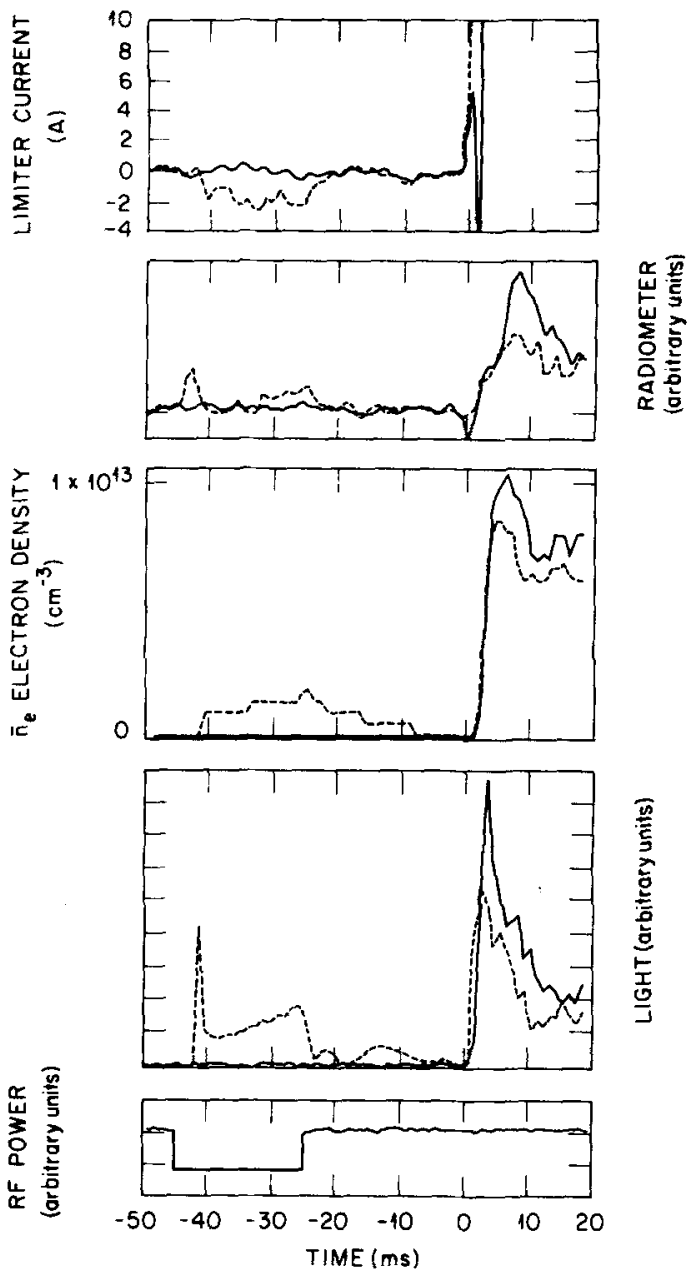

FIG. 8. Several diagnostic signals as a function of time with (dotted lines) and without (solid lines) an early 20 -msec preionization pulse starting 45 msec before capacitor bank firing. Shown are the visible light from a pin diode, density (horizontal 2-mm interferometer), current to upper limiter, and a wide-angle radiometer signal. Note the reduction of all signals by the preionization pulse and the peculiar behavior of the light signal after if turnoff.

$\mathrm{keV}$ could be contained in this way by a 5-G vertical error field, and calculations suggest that a small toroidal current below the threshold of detectability could be responsible for the observations. On the other hand, Stix ${ }^{14}$ and Bulyginskii et al. ${ }^{15}$ have proposed mechanisms for confinement of plasma in purely toroidal fields by means of a space potential buildup that provides an effective rotational transform. The possibility that an ambipolar potential aids confinement has also been discussed by Borowski et al. ${ }^{16}$

After the capacitor firing, other features are of interest. All of the signals show an effect from the preionization pulse. The loop voltage (not shown) is lower, the density is reduced from the value achieved without microwave power, and the light spike and the radiometer spike are reduced. These changes are further evidence that the early preionization pulse has altered the conditions inside the vacuum chamber.

Power should be deposited on the limiters during preionization because of curvature $(B \times \nabla B)$ drift and field error drift. An infrared camera was oriented to look at the upper limiter through a CaF window by means of a frontsurface gold mirror. No limiter surface temperature rise could be detected, even though the limiter current was sever- 
al amperes. However, only about half the length of the limiter could be viewed through the window, and it was not certain that the heating zone was positioned under that section.

The double floating probe located in the shadow of the upper limiter was used to measure the temperature and density of the preionized plasma during this experiment. The probe gave an electron temperature of $2.4 \mathrm{eV}$ and a density of $1.3 \times 10^{12} \mathrm{~cm}^{-3}$. It was located just above the upper limiter and separated by three sectors $\left(120^{\circ}\right)$ toroidally. The probe was at a major radius of $95.25 \mathrm{~cm}$, or $2.25 \mathrm{~cm}$ beyond the cyclotron resonant layer at $93 \mathrm{~cm}$. For a plasma density of $1.3 \times 10^{12} \mathrm{~cm}^{-3}$, the upper hybrid layer would be at a major radius of about $100 \mathrm{~cm}$. Hence, the probe would not be positioned over this layer, and the temperature and density measured would characterize not this layer but rather the plasma nearer the cyclotron resonance. The density is in fair agreement with the line density measured by the interferometer. Based on the results of Ref. 1, the temperature near the upper hybrid layer could be considerably larger than that measured by the probe.

A VUV monochromator viewed the plasma across the midplane. In principle, it is possible to measure the plasma electron temperature by measuring the intensities of a number of impurity lines, as was done in Ref. 1. However, in the current experiments the signals were very noisy, and it was not possible to make a determination of the electron temperature by this method.

\section{F. Reduction of capacitor bank voltages with a constant 5-msec preionization pulse}

In a study that is of interest for future large tokamaks, ${ }^{16,17}$ the capacitor bank voltages on the inner and outer supplies were lowered together so that the toroidal current would start up approximately centered and remain there. The outer bank was lowered from its typical value of 220 to $100 \mathrm{~V}$, and the inner bank voltage was lowered from 100 to $40 \mathrm{~V}$. With only these lowered voltage capacitor banks supplying power, the plasma created was very resistive and the current buildup was slow and short-lived (35-msec duration). However, with the application of preionization from -5 to +6 msec relative to the capacitor bank firing, the discharge duration was extended to $90 \mathrm{msec}$. The plasma current was actually detectable $4 \mathrm{msec}$ earlier with the preionization. The longer discharge used the same amount of transformer flux (volt-seconds) as the short-lived discharge. Figure 9 shows the temporal behavior of the plasma current, loop voltage, density, and if pulse timing for the first $40 \mathrm{msec}$ of the discharge.

Current start-up with much lower voltages makes possible a considerable saving in power supply costs for large tokamaks. Also, the poloidal field system can be simplified and the inner wall can be made more conductive for added protection of the poloidal circuits during disruptions.

\section{G. Evidence of field errors}

There were no error field correction coils on ISX-B for the purpose of changing or eliminating stray magnetic fields. There were two ways to examine field errors. One was to
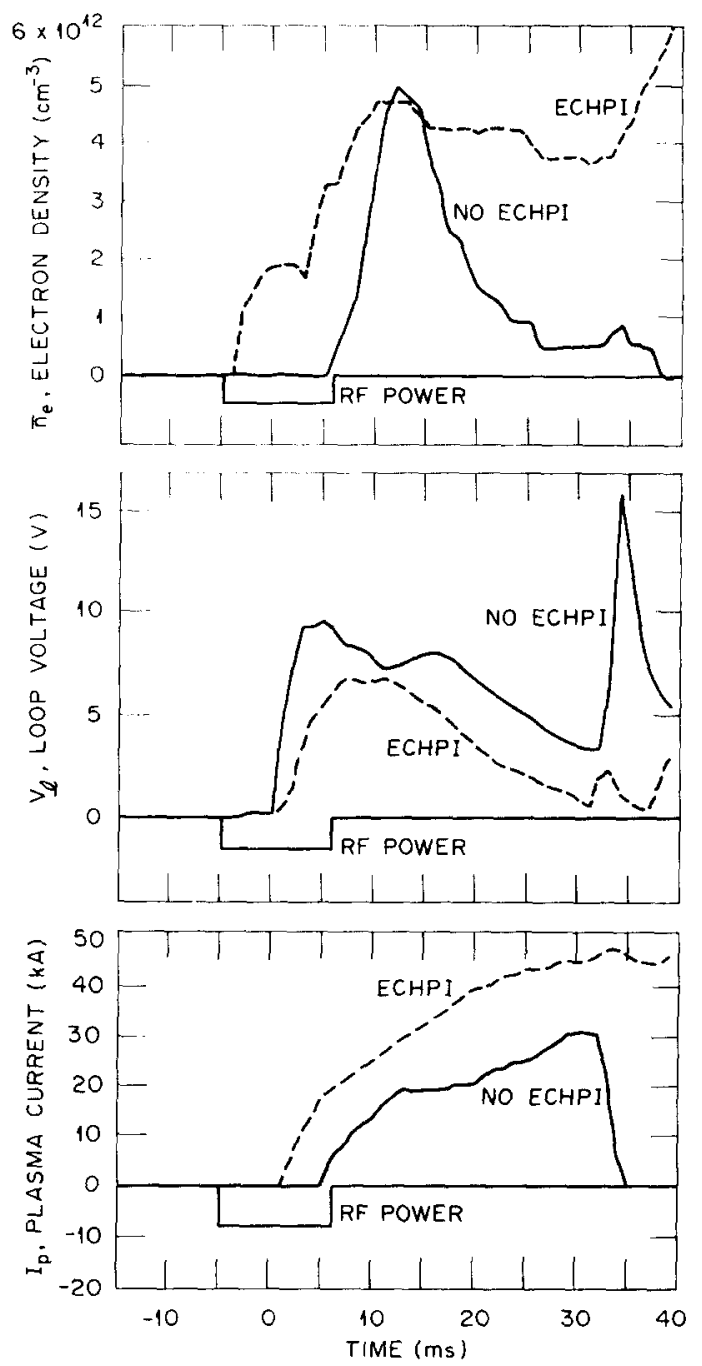

FIG. 9. Temporal variation of the electron density, loop voltage, plasma current, and microwave power for two discharges with lowered capacitor bank voltages, one with (dotted lines) and one without (solid lines) preionization. Only the first $40 \mathrm{msec}$ of the discharge is shown. The plasma duration for the case with preionization is $90 \mathrm{msec}$, the density rises to $\simeq 2 \times 10^{13}$ $\mathrm{cm}^{-3}$, and the current reaches a plateau of $50 \mathrm{kA}$. Note that the current is detectable about 4 msec earlier with preionization than without it.

reverse the direction of the toroidal field, which would change the curvature drift direction. In the absence of field errors, this reversal would only change the sign of the limiter current and not its magnitude. The other was to use the inner and outer windings to generate a field without a tokamak discharge.

Figure 10 shows the upper limiter current due to a preionized plasma with a constant microwave pulse power and timing with the two magnetic field directions. All other conditions are held constant. Note that both the sign and magnitude change; the latter change indicates the presence of an error field. However, it is impossible to determine the size of the error field from this.

Other evidence suggested that accidental error fields produced strong limiter currents in the presence of microwave plasma production. These fields were produced by capacitor discharges in the inner and outer windings. However, it was not possible to calculate from these data the magnitude of the actual error field without these accidental fields. 


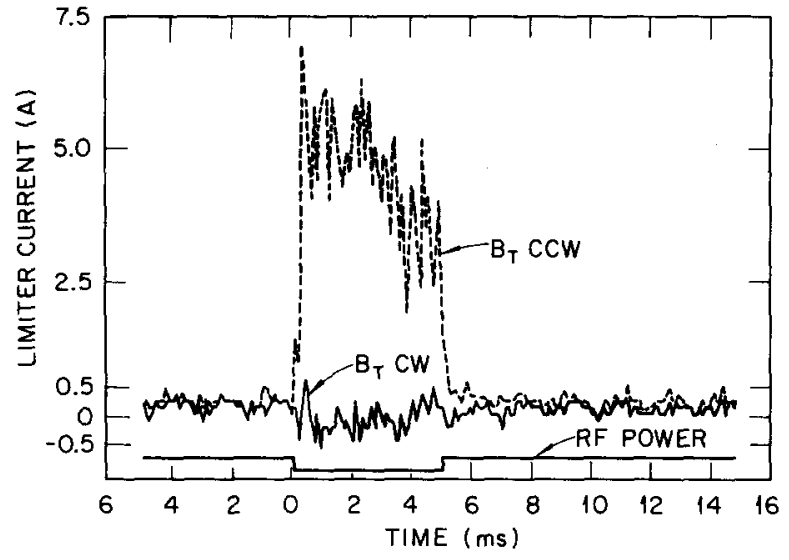

FIG. 10. Upper limiter current with a constant preionization pulse and with both toroidal field directions, clockwise and counterclockwise. The lower trace shows the microwave timing.

Calculations ${ }^{18}$ have shown that there is probably a vertical error field of $5 \mathrm{G}$ due to the back bias winding on the iron core. Also, there is a radial error field from the toroidal bus bars. These bus bars have a horizontal separation, and the quadrupole component of this field gives a radial error field of $8 \mathrm{G}$ at the center of the plasma. The fractional field error from these two effects is $\delta B / B \simeq 1 \times 10^{-3}$. Further discussion of the effects of error fields is found in Sec. IV C.

\section{ZERO-DIMENSIONAL MODEL OF PREIONIZATION}

A zero-dimensional model of the preionization by $\mathrm{ECH}$ is used to compare with the experiment and to develop scaling predictions. This model is based on the work of Holly $e t$ $a l .^{6}$ but has rather major modifications. Specifically, it has an added ECH term and an error field loss term, and the discharge is no longer considered to be neutral dominated. The model of Fontenesi et al. ${ }^{19}$ does not include field errors and, as a consequence, predicts densities and electron temperatures somewhat higher than those produced by our model and higher than those observed in the experiment of Ref. 1.

The first $10 \mathrm{msec}$ or so of the discharge are modeled, so that processes such as the transfer of energy to the ions from the electrons are neglected. The typical timing includes a preionization pulse followed by an ohmic heating phase; the two may overlap. The quantities used are the electron density, the electron energy density, the neutral density, the electron temperature, the plasma current, and the loop voltage of the tokamak. Each of the densities is treated as a volume average, and quadratic radial dependences are assumed when converting to line averages or peak values. In contrast to the work by Peng et al., ${ }^{9}$ this model does not include the possible processes of electron heating near the upper hybrid resonance and electron confinement by ambipolar potential near the metallic vessel wall.

We believe that the most serious deficiency in the model is geometrical. That is, one does not know the size and shape of the plasma or the position of the microwave heating surface. If the heating occurs at cyclotron resonance in the center of the chamber, the plasma containment may be quite different from the containment of a plasma heated at the upper hybrid resonance at the outer edge of the machine. In the model we assume a torus with minor radius $a$ taken to be the actual machine dimension.

\section{A. The equations}

\section{The electron density}

The electron density $n_{e}$ is governed by the rate equation

$$
\frac{d n_{e}}{d t}=n_{e} v_{\text {ion }}-n_{e}\left(v_{\text {err }}+v_{d r}+v_{\text {dif }}\right),
$$

with

$$
v_{\text {ion }}=n_{0}\langle\sigma v\rangle_{\text {ion }} .
$$

The ionization rate is taken from published data. ${ }^{20}$ The firstorder field error causes field lines and plasma to spiral out of the machine, with the electrons moving at their thermal velocity $v_{T}=\left(T_{e} / m\right)^{1 / 2}$. The distance traveled is the minor radius $a$ divided by the angle that the actual field line makes with the ideal field line, $\delta B / B$. With a finite rotational transform, the field error loss becomes a random walk, which is negligible. The crossover where the direct loss becomes a random walk occurs at a critical current $I_{c}$, defined as the current where the poloidal magnetic field equals the error field at the edge of the plasma, $I_{c}=4 \pi a \delta B / \mu_{0}$. The field error loss rate is

$$
v_{\text {err }} \simeq(\delta B / B)\left(v_{T} / a\right)\left[1-\left(I_{p} / I_{c}\right)\right]
$$

for $I_{p}<I_{c}$ and zero for $I_{p}>I_{c}$.

The loss from the curvature and gradient $B$ drift is also only present with negligible rotational transform and is treated in the same way. A troublesome problem arises here, since the opposite directions of the electron and ion drifts lead to plasma polarization and to a polarization drift ${ }^{21}$ of unknown magnitude. One experiment has led to the conclusion that the plasma drifts outward at a rate near the ion sound velocity. ${ }^{22}$ In contrast, Peng et al. ${ }^{9}$ have suggested that the loss is determined by the potential drop across the sheath at the plasma boundary. We assume that the loss is given by the vertical drift, since this rate is $R / a$ times the polarization loss rate if the average potential is $V=T / e$. We use

$$
v_{d r}=\left(2 T / a R \omega_{c} m\right)\left[1-\left(I_{p} / I_{c}\right)\right],
$$

where $\omega_{c}$ is the electron cyclotron frequency and $R$ is the major radius of the tokamak. This loss rate is also zero for $I_{p}>I_{c}$.

The diffusive loss is produced by diffusion from classical electron-ion and electron-neutral collisions:

$$
v_{\mathrm{diff}}=8 T_{e}\left(v_{e i}+v_{e o}\right) / m \omega_{c}^{2} a^{2} .
$$

This rate is usually negligibly small.

\section{The neutral density}

The basic problem asociated with treating the neutral density is the treatment of recycling; just how many neutrals leave the wall when it is struck by an electron or ion? Here we assume that the time scale for recycling is longer than the other time scales in the problem and that neutrals are lost at the same rate at which electrons are produced, which is equivalent to a recycling coefficient of one. Best estimates of the recycling coefficient give values near one. ${ }^{23}$ We assume

$$
\frac{d n_{0}}{d t}=-\frac{d n_{e}}{d t} \text {. }
$$




\section{The energy density}

The volume-averaged energy density is $U=3 n_{e} T_{e} / 2$, which includes only the electron energy, since the ion energy is very small early in the discharge. The energy density rate equation is

$$
\frac{d U}{d t} \simeq \frac{P_{\mathrm{ECH}}+P_{\mathrm{OH}}-P_{\mathrm{rad}}}{V_{\mathrm{pl}}}-U\left(v_{\mathrm{err}}+v_{d r}+v_{E}\right),
$$

where all quantities are volume averaged and $V_{\mathrm{pl}}$ is the plasma volume. The entire volume of the tokamak is usually used, although the ECH power may be deposited in a smaller volume.

The principal power source is the ECH power $P_{E C H}$. With input power $P_{0}$, the (single-pass) absorbed power at moderate densities and temperatures is ${ }^{24}$

$$
P_{\mathrm{ECH}}=P_{0}\left[1-f_{o} \exp \left(-\eta_{0}\right)-f_{x} \exp \left(-\eta_{x}\right)\right],
$$

where $\eta_{0}$ and $\eta_{x}$ are the dimensionless optical depths, and $f_{o}$ and $f_{x}$ are the fractional powers for the ordinary and extraordinary modes, respectively.

In the range of temperatures and densities that occur in preionization experiments, the optical depths have not been calculated or measured. In the extremely low density limit, where the plasma dispersion can be neglected and the cutoff layer of the extraordinary mode is very thin, the optical depths are the same ${ }^{25}$

$$
\eta_{x}=\eta_{o}=\left(\pi^{2} / 2\right) \alpha(R / \lambda),
$$

where $\alpha=\omega_{p}^{2} / \omega^{2}$ and $\lambda$ is the free space wavelength of the incident wave. At higher density, the heating by the extraordinary mode occurs at the upper hybrid surface through a nonlinear mode conversion. ${ }^{9}$ In both cases the absorption is predicted to be essentially complete. Therefore we assume the optical depth

$$
\eta=\left(\pi^{2} / 2\right) \alpha(R / \lambda)(1-\alpha)^{2},
$$

where the added factor cuts off the heating for $\alpha \geqslant 1$. This formula yields complete absorption below cutoff for tokamak parameters. In the simulations, the cutoff only occurred during the ohmic heating phase.

The radiation loss $P_{\text {rad }}$ is produced entirely by the ionization process. With each ionization event an energy $E_{i}=30 \mathrm{eV}$ is lost, so

$$
P_{\mathrm{rad}}=E_{i} v_{\text {ion }} \text {. }
$$

The ohmic heating power is

$$
P_{\mathrm{oh}}=I_{p}^{2} R_{\mathrm{pl}} \text {, }
$$

where $I_{p}$ is the plasma current and $R_{\mathrm{pl}}$ the plasma resistance, with

$$
R_{\mathrm{pl}}=2 R / a^{2} \sigma_{e},
$$

and $\sigma_{e}$ the electrical conductivity. The conductivity is calculated with both electron-ion and electron-neutral collisions. The conductivity from Coulomb collisions is taken from Ref. 26, and for the neutral collisions we use

$$
\sigma=\frac{\omega_{p}^{2}}{4 \pi \sigma_{e 0} n_{0}\left(T_{e} / m\right)^{1 / 2}},
$$

where the electron neutral cross section is taken to be $\sigma_{\mathrm{e} 0}=5.0 \times 10^{-15} \mathrm{~cm}^{2}$.
The convective loss rates from the error field and the magnetic drift are the same as for the density, but the basic energy loss when the current is more than the critical value is the empirical scaling, "twice Alcator scaling," with an energy containment time

$$
\tau_{E}=\text { const } n_{e} a^{2},
$$

and

$$
v_{E}=1 / \tau_{E},
$$

when $I_{p}>I_{c}$.

\section{The current}

The electrical circuitry is treated by a primitive model with a constant applied voltage $V$, a constant inductance $L=1.1 \mu \mathrm{H}$, and the calculated resistance

$$
V=L \frac{d I_{p}}{d t}+I_{p} R_{\mathrm{pl}} \text {. }
$$

These four equations were solved by standard integration techniques (e.g., Runge-Kutta and Adams methods). All methods used gave the same temporal evolution of the electron temperature and density and the neutral density.

\section{B. Results of the calculations}

The time history of a typical simulation is shown in Fig. 11. Figure 11(a) shows the electron density $n_{e}$ and the electron temperature $T_{e}$. The ECH is applied for $6 \mathrm{msec}$ starting at $t=0$ and the ohmic heating is started at $5 \mathrm{msec}$ (the sequence commonly used in the experiment). The initial neutral density is $n_{0}=7 \times 10^{12} \mathrm{~cm}^{-3}$, corresponding to a fill pressure of $\simeq 1 \times 10^{-4}$ Torr. The applied voltage is $20 \mathrm{~V}$, the ECH power is $70 \mathrm{~kW}$, and the fractional field error $\delta B / B$ is $1.5 \times 10^{-3}$. The $\mathrm{ECH}$ frequency is $28 \mathrm{GHz}$, and the volumeaveraged cutoff density is $5 \times 10^{12} \mathrm{~cm}^{-3}$.

The electron density rises to $2.2 \times 10^{12} \mathrm{~cm}^{-3}$, in agreement with the experimental results for preionization, and

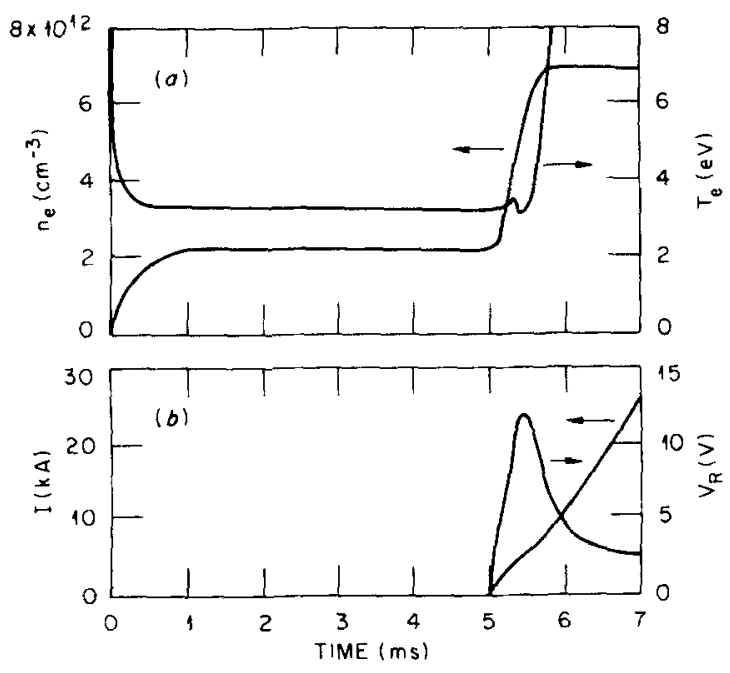

FIG. 11. Plots of (a) the electron density $n_{e}$ and temperature $T_{e}$ and (b) the toroidal current $I$ and the resistive loop voltage $V$, as functions of time from the model. The microwave power is applied in the interval 0 to $6 \mathrm{msec}$, and the ohmic heating is applied at $t=5 \mathrm{msec}$. The parameters are $V=20 \mathrm{~V}$, $P_{0}=70 \mathrm{~kW}, \delta B / B=1.5 \times 10^{-3}, f=28 \mathrm{GHz}, n_{0}=7.0 \times 10^{12} \mathrm{~cm}^{-3}$, $T(0)=1 \mathrm{eV}, n_{e}(0)=1.0 \times 10^{12} \mathrm{~cm}^{-3}, R=93 \mathrm{~cm}$, and $a=27 \mathrm{~cm}$. 
then rises again with the ohmic heating to its maximum value given by the gas fill. The electron temperature, after an initial peak, decays to an equilibrium value of $3.3 \mathrm{eV}$ with preionization only. The electron temperature rises again with the ohmic heating. This behavior is in general agreement with the experimental results, although the electron temperature is not measured at early times on this short a time scale. The temperature calculated here is somewhat higher $(30 \%)$ than that measured with the double floating probe (Sec. III E).

With smaller field errors (e.g., $\delta B / B=1 \times 10^{-4}$ ) the density rises to the cutoff density, $5 \times 10^{12} \mathrm{~cm}^{-3}$. This does not correspond to the experimental results.

Figure 11 (b) shows the toroidal current $I_{p}$ and the resistive part of the loop voltage $V_{r}$. The current rises at a rate of $14 \mathrm{kA} / \mathrm{msec}$ at $1 \mathrm{msec}$ after the ohmic heating is turned on. This is somewhat above the experimental value. With no preionization, the value of $d I_{p} / d t$ is about $7 \mathrm{kA} / \mathrm{msec}$, which is close to the experimental value.

A study of the ECH absorbed power and the radiated power shows why the electron temperature remains so low during preionization. The ionization completely dominates the energy balance. A slight increase in $T_{e}$ increases the ionization cross section, which increases the number of electrons, and the associated radiative energy loss limits the temperature. As a consequence, the radiated power is almost equal to the input power. The burst of radiation associated with the beginning of the ohmic heating is observed experimentally and is limited by the depletion of the neutral density. For the case of start-up with no preionization there is also a burst of radiation but of much longer duration. It is also limited by the depletion of the neutral density. This too is in rough agreement with the experimental observation. A more realistic model for the radiated power would include the radiation by impurity atoms, ${ }^{26}$ which can provide an even more severe energy loss. However, with the electron temperature limited to a few electron volts by the ionization process, the impurity atoms should not be highly excited.

\section{Parameter variation}

In order to evaluate changes in parameters, we chose to examine the electron density and temperature at the end of the ECH pulse (just before the ohmic heating comes on), the resistive loop voltage $V_{r}$ at $0.6 \mathrm{msec}$ after the ohmic heating is applied ( $5.6 \mathrm{msec}$ into the simulation), and $d T_{p} / d t$ evaluated $1 \mathrm{msec}$ after the ohmic heating is applied $16 \mathrm{msec}$ into the simulation). These times are picked to correspond to the times used in the experimental evaluation in Sec. III.

These quantities are shown in Fig. 12 as a function of the fractional field error, $\delta B / B$. The initial increase in $T_{e}$ is caused by the decrease in the density and hence the total number of electrons in the machine with a constant power input.

The resistive loop voltage decreases slightly with increasing $\delta B / B$ (below $8 \times 10^{-4}$ ) due to the increasing conductivity. However, at larger field errors the loop voltage rises due to the much lower density and hence lower conductivity. The value of $d I_{p} / d t$ remains high for small field errors and eventually drops to near zero for very large field errors.
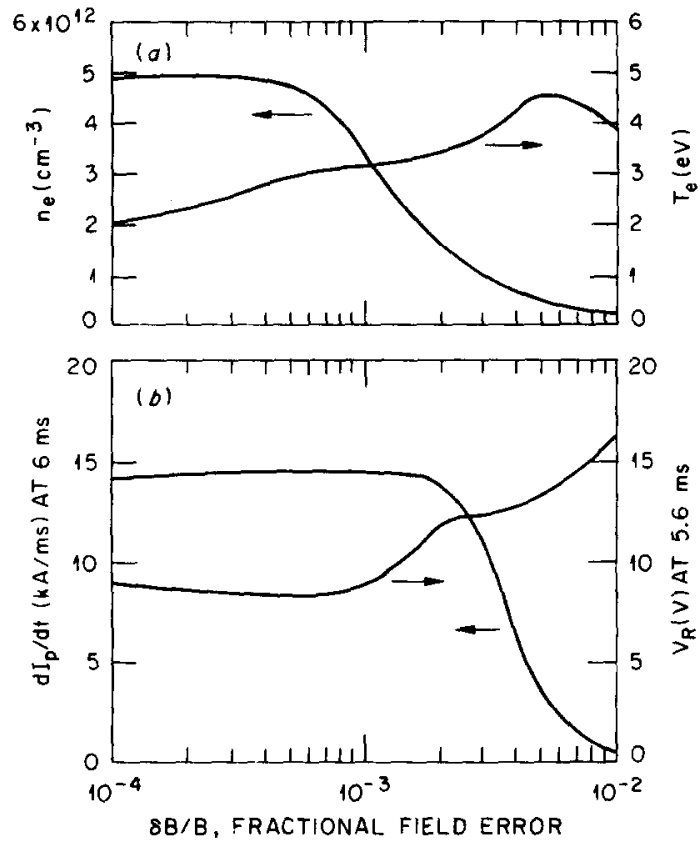

FIG. 12. (a) The electron density $n_{e}$ and temperature $T_{e}$ at $5 \mathrm{msec}$, plotted as a function of the field error $\delta B / B$. The other parameters are those of Fig. 11. (b) The current rate of rise $d I_{p} / d t$ at $6 \mathrm{msec}$ and the resistive voltage $V$, at $5.6 \mathrm{msec}$, plotted as functions of $\delta B / B$ with the parameters of Fig. 11 .

The current will not start for $\delta B / B>10^{-2}$. By comparison with the experimental data, we judge that the actual fractional field error is $1-2 \times 10^{-3}$ or $\delta B=10-20 \mathrm{G}$. This is in satisfactory agreement with the value found in Sec. III G.

The variation of various parameters as a function of ECH power is shown in Fig. 13. The relative field error is taken as $1.5 \times 10^{-3}$. With a field error of this size the electron density rises linearly with power, in agreement with experiment. The calculated density at $70 \mathrm{~kW}$ closely corresponds to the actual density observed. The electron temperature is only weakly dependent on the power, due to the aforementioned arguments about radiative power loss. We note that at the gas densities typical of this experiment, it is almost impossible to get a very high electron temperature. For this field error, a power of $1 \mathrm{MW}$ gives $T_{e}=4.5 \mathrm{eV}$. The value of $d I_{p} / d t$ is almost constant over the range of 30 to 70 $\mathrm{kW}$, which was the range of power used in the experiment. This corresponds to the experimental observation. However, the drop in the resistive loop voltage is greater in the simula-

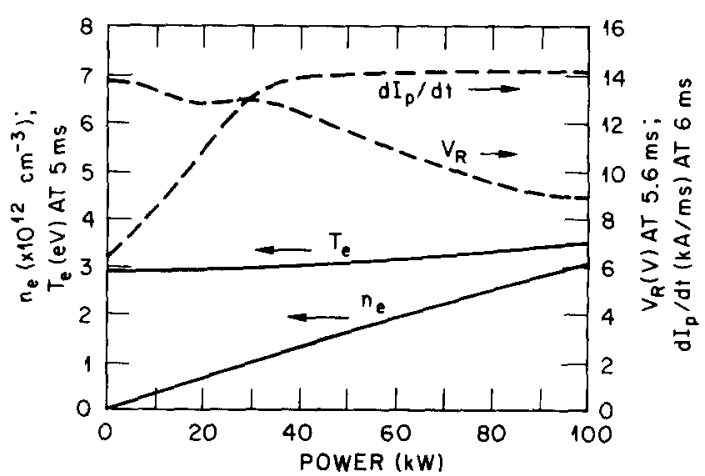

FIG. 13. The electron density $n_{e}$ and temperature $T_{\varepsilon}$ at $5 \mathrm{msec}$, plotted as functions of the microwave power $P_{\mathrm{ECH}}$ for the model. The other parameters are those of Figs. 11 and 12. Also plotted are $d I_{p} / d t$ at $6 \mathrm{msec}$ and $V$, at $5.6 \mathrm{msec}$. 
tion than was observed experimentally. In contrast, with a very small field error, the electron density rises in the simulation very quickly to the cutoff value at low power.

Figure 14 shows the variation of $n_{e}$ and $T_{e}$ with gas density at a constant power input and a fractional field error of $1.5 \times 10^{-3}$. The density increases monotonically with pressure in agreement with experimental observations. The scaling study shows that $T_{e}$ would rise to much higher values at low gas density, $d I_{p} / d t$ would also increase, and $V_{r}$ would sharply decrease. These quantities were not measured in the experiment, suggesting another interesting area for further experimentation. Smaller field errors $\left(\leqslant 5 \times 10^{-4}\right)$ give a saturation of $n_{e}$ at the cutoff density, contrary to the experimental observations.

The decay time of the plasma density with no ohmic heating was measured a number of times in the experiment and found to be approximately $10 \mathrm{msec}$. Clearly, long-term confinement of the plasma existed. Furthermore, the loop voltage was affected for preionization occurring as much as $30 \mathrm{msec}$ before the onset of ohmic heating. These effects could not be reproduced in the model. Only for error fields of less than $6 \times 10^{-5}$ could the $e$-folding time of the density decay reach $10 \mathrm{msec}$, which is in disagreement with the other observations. The $e$-folding time $\tau$ in our model was found to be related to the error field by the relation

$$
\tau=6 \times 10^{-7}(\delta B / B)^{-1} \text {. }
$$

Similarly, a study of $d I_{p} / d t$ and $V_{r}$ with a delay in the ohmic heating turn-on showed that these quantities do not follow their experimentally determined values. In the model with $\delta B / B=10^{-3}$, the value of $d T_{p} / d t$ drops to its nonpreionized value within a few milliseconds after preionization turn-off and the value of $V_{r}$ rises to its nonpreionized value with the same time scale.

The variation in $\tau$ with gas pressure was less pronounced. There was only an $18 \%$ change in $\tau$ as the gas pressure was varied from $3 \times 10^{12}$ to $12 \times 10^{12} \mathrm{~cm}^{-3}$.

It is clear from these results that the decay of the plasma in the actual device cannot be reproduced by our model under reasonable assumptions and hence is probably governed by other processes (such as those proposed by Peng et al. ${ }^{9}$ ) or by a superthermal toroidal current. Although other refinements can be made to the present model to make the results

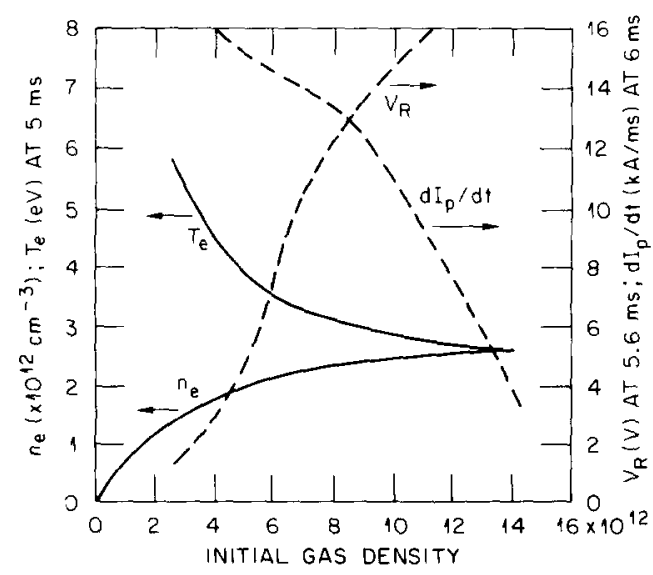

FIG. 14. The density $n_{e}$ and temperature $T_{e}$ at $5 \mathrm{msec}$, plotted as functions of the initial neutral density $n_{0}$ from the model. Also plotted are $d I_{p} / d t$ at 6 msec and $V_{r}$ at $5.6 \mathrm{msec}$. The other parameters are those of Figs. 11-13. more closely agree with the present data, the model stands as a reasonable approximation to the actual conditions during the preionization, excluding the decay process. We also note that since this is a zero-dimensional model we cannot predict the experimental results of Figs, 4 and 5. These figures show the effect of varying the magnetic field, which moves the resonance region through the tokamak volume. Furthermore, although the limiter current measurements were instrumental in an analysis of the error fields, these currents are not included in the model.

\section{Extrapolation to higher frequencies, larger devices, and higher fields}

The model has been applied to the original preionization experiment in ISX-B using $80 \mathrm{~kW}$ of $35-\mathrm{GHz}$ power. ${ }^{1}$ Generally, conditions under which a density of $5 \times 10^{12}$ $\mathrm{cm}^{-3}$ was achieved were easily obtained, but temperatures were typically a few electron volts, as in the $28-\mathrm{GHz}$ experiment. Two points should be made. The $35-\mathrm{GHz}$ experiment was performed in 1979 prior to some changes in the toroidal field bus work. These bus-work changes resulted in an increase in the radial error field to approximately $8 \mathrm{G}$ as noted in Sec. III G. However, in 1979 the currents in the original bus work would have produced roughly half this error field. Hence, the total fractional field error from all sources should have been somewhat less. We estimate that $\delta B / B$ $=5 \times 10^{-4}$ to $1 \times 10^{-3}$. The second point is that for the specific experiment in Ref. 1 in which the electron temperature was measured, the line density was $2.2 \times 10^{12} \mathrm{~cm}^{-3}$. Typical bulk temperatures of 8-13 eV were obtained by a scanning gridded probe, while regions of higher temperature were observed near the calculated position of the upper hybrid resonance.

Using a lower gas pressure of approximately $3 \times 10^{12}$ $\mathrm{cm}^{-3}$, the model gives a density of $2.5 \times 10^{12} \mathrm{~cm}^{-3}$ and a temperature of $14 \mathrm{eV}$ for a fractional field error of $5 \times 10^{-4}$. The increased electron temperature is due to the reduction in gas pressure. Under these conditions, the radiated power is much less than in our standard $28-\mathrm{GHz}$ case with full gas feed. However, we could not reproduce the result in Ref. 1 in which it was determined that only approximately $5 \mathrm{~kW}$ out of $80 \mathrm{~kW}$ was actually radiated.

The present model has also been applied to the current experiments on the Poloidal Divertor Experiment (PDX) and Doublet III (DIII), which both use 60-GHz gyrotrons. Plans are to use $400 \mathrm{~kW}$ of power on $\mathrm{PDX}^{27}$ and to eventually have $2 \mathrm{MW}$ of power on DIII. ${ }^{28}$ At present, each one has two gyrotrons for a maximum power of $420 \mathrm{~kW}$. Both devices have approximately the same major and minor radii, which are taken here as 143 and $44 \mathrm{~cm}$, respectively.

For the cases with $400 \mathrm{~kW}$, the model predicts that the volume-averaged density would reach $6 \times 10^{12}$ with $T_{e} \simeq 4.5 \mathrm{eV}$ for a fractional field error of $1 \times 10^{-3}$. For the higher power typical of DIII, the value of the density will rise to the limit set by the gas feed, but the temperature can rise significantly. For $1 \mathrm{MW}, T_{e}$ would reach $12.8 \mathrm{eV}$; for $2 \mathrm{MW}$, $T_{e}$ would reach $25.9 \mathrm{eV}$. Again, this high temperature is due to the low gas pressures assumed with this higher frequency source so that the density cannot reach the cutoff density. If 
preionization is attempted with these parameters, breakdown should be facilitated.

\section{SUMMARY AND CONCLUSIONS}

A plasma was produced in the ISX-B tokamak using microwave power from a $28-\mathrm{GHz}$ gyrotron at the electron cyclotron resonance. The influences of the toroidal field, gas pressure, microwave power, pulse length, timing, and error fields were studied. Both pure microwave discharges and discharges that were initiated with microwaves and followed by ohmic heating were studied. In the latter discharges, the effect of the capacitor bank voltage was also studied.

It was found that the optimum toroidal field was that which displaced the resonance toward the outside. The optimum pulse length was found to be $5 \mathrm{msec}$, but large effects were found for much shorter pulses. Timing that overlapped the main discharge was best, but early pulses had a large effect. With preionization, much lower applied voltages could be used.

A theoretical model that adequately reproduces the characteristics of the discharges has been developed for both 28- and $35-\mathrm{GHz}$ plasma production. The decay of the afterglow discharge, with no power input, is not correctly treated by this model. Some other physical process maintains the very low density, low-temperature plasma for a longer time than predicted by the present model. However, this model is adequate for the start-up phase of tokamaks, where the microwave and ohmic heating power dominates. Calculations based on this model show the temporal evolution of the electron temperature and density and the plasma current. One significant conclusion is that the magnitude of the error field is critically important. Further calculations show that this preionization technique will be useful in other devices at higher magnetic field strengths.

\section{ACKNOWLEDGMENTS}

The support and encouragement of M. J. Saltmarsh and J. Sheffield are gratefully acknowledged. The authors also acknowledge discussions and technical contributions from S. C. Bates, S. M. DeCamp, J. L. Dunlap, G. R. Dyer, J. C. Ezell, W. A. Gabbard, D. J. Hoffman, R. C. Isler, T. C. Jernigan, H. E. Ketterer, J. F. Lyon, L. E. Murray, M. Murakami, G. H. Neilson, J. Pierce, and W. R. Wing.

This work was supported by the Office of Fusion Energy, U. S. Department of Energy, under Contract No. W7405-eng-26 with the Union Carbide Corporation.

'R. M. Gilgenbach, M. E. Read, K. E. Hackett, R. F. Lucey, V. L. Granatstein, A. C. England, C. M. Loring, J. B. Wilgen, R. C. Isler, Y-K. M.
Peng, K. H. Burrell, O. C. Eldridge, M. P. Hacker, P. W. King, A. G. Kulchar, M. Murakami, and R. K. Richards, Nucl. Fusion 21, 319(1981). ${ }^{2}$ D. G. Bulyginskii, M. M. Larionov, L. S. Levin, O. V. Mikluckho, A. I. Tokunov, and N. V. Shustova, Sov. J. Plasma Phys. 6, 11 (1980).

${ }^{3}$ K. Ohkubo, K. Kawahata, K. Matsuoka, N. Noda, K. Sakurai, S. Tanahashi, J. Fugita, M. Tanaka, H. Hosokawa, M. Fujiwara, H. Ikegami, T. Cho, Y. Terumichi, and S. Tanaka, Nucl. Fusion 21, 1320 (1981).

${ }^{4}$ T. Cho, S. Kubo, M. Ikeda, T. Saito, Y. Terumichi, Y. Hamada, and S. Tanaka, Phys. Lett. A 77, 318 (1980).

${ }^{5}$ Y. Terumichi, T. Maekawa, T. Shimozuma, T. Saito, M. Nakamura, T. Cho, S. Kubo, Y. Hamada, and S. Tanaka, in Proceedings of the 4th Topical Conference on RF Heating in Plasmas (University of Texas, Austin, 1981), Paper D3.

${ }^{6}$ D. J. Holly, S. C. Prager, D. A. Shepard, and J. C. Sprott, Nucl. Fusion 21, 1483 (1981).

${ }^{7}$ N. Suzuki, in Proceedings of the European Physical Society, Moscow, September 1981 (European Physical Society, Moscow, 1981), Vol. 5H, p. 307.

${ }^{8}$ A. 1. Anisomov, N. I. Vinogradov, and B. P. Poloskin, Sov. Phys.-Tech. Phys. 18, 459 (1973); 20, 626 (1976); 20, 629 (1976).

${ }^{9}$ Y-K. M. Peng, S. K. Borowski, and T. Kammash, Nucl. Fusion 18, 1489 (1978).

${ }^{10}$ M. Murakami, D. W. Swain, S. C. Bates, C. E. Bush, L. A. Charlton, J. L. Dunlap, G. R. Dyer, P. H. Edmonds, A. C. England, H. H. Haselton, D. P. Hutchinson, R. C. Isler, T. C. Jernigan, S. Kasai, H. E. Ketterer, J. Kim, P. W. King, E. A. Lazarus, J. F. Lyon, C. H. Ma, J. T. Mihalczo, J. K. Munro, A. P. Navarro, G H. Neilson, D. R. Overbey, V. K. Paré, M. J. Saltmarsh, S. D. Scott, J. Sheffield, J. E. Simpkins, W. L. Stirling, C. C. Tsai, R. M. Wieland, J. B. Wilgen, W. R. Wing, R. E. Worsham, and B. Zurro, Plasma Physics and Controlled Nuclear Fusion Research, Brussels, 1980 (IAEA, Vienna, 1981), Vol. I, p. 390.

${ }^{11}$ R. D. Wengenroth, IEEE Trans. Microwave Theory Tech. MTT-26, 332 (1978).

${ }^{12}$ K-L. Wong, R. Horton, and M. Ono, Phys. Rev. Lett. 45, 117 (1980).

${ }^{13} \mathrm{M}$. Nakamura, S. Suzuki, Y. Terumichi, Y. Hamada, and S. Tanaka, Phys. Lett. A 81, 383 (1981).

${ }^{14}$ T. H. Stix, Phys. Fluids 14, 692 (1971); 14, 702 (1971).

${ }^{15}$ D. G. Bulyginskii, V. S. Yuferev, and E. V. Galaktionov, Sov. J. Plasma Phys. 3, 528 (1977).

${ }^{16}$ S. K. Borowski, Y-K. M. Peng, and T. Kammash, Nucl. Technol./Fusion (in press).

${ }^{17}$ A. G. Kulchar, O. C. Eldridge, A. C. England, C. M. Loring, P. H. Edmonds, and M. Murakami, Proceedings of the 1981 IEEE International Conference on Plasma Science (IEEE, New York, 1981), p. 142.

${ }^{18} \mathrm{G}$. H. Neilson (private communication).

${ }^{19}$ M. Fontinesi, C. Maroli, V. Petrillo, and A. Partani, Proceedings of the 1982 International Conference on Plasma Physics, Goeteborg, Sweden, 1982, p. 23; M. Fontinesi, C. Maroli, and V. Petrillo, Heating in Toroidal Plasma: Proceedings of the 3rd Joint Varenna-Grenoble International Symposium, 1982 (Commission of the European Communities, Brussels, 1982), Vol. II, p. 699.

${ }^{20}$ R. L. Freeman and E. M. Jones (private communication).

${ }^{21}$ L. Spitzer, Physics of Fully Ionized Gases (Interscience, New York, 1962), Chap. 1.

${ }^{22} \mathrm{~J}$. C. Sprott (private communication).

${ }^{23}$ P. K. Mioduszewski (private communication).

${ }^{24}$ O. C. Eldridge, W. Namkung, and A. C. England, Oak Ridge National Laboratory Report No. ORNL/TM-6052, 1977.

${ }^{25}$ O. C. Eldridge, Phys. Fluids 15, 676 (1972).

${ }^{26}$ D. L. Book, NRL Plasma Formulary (Naval Research Laboratory, Washington, D. C., 1978), p. 30.

${ }^{27}$ H. Hsuan, Princeton Plasma Physics Laboratory (private communication).

${ }^{28}$ R. Prater, C. P. Moeller, S. H. Lin, the Doublet III Physics Group, K. Hoshino, and the JAERI Physics Group, Proceedings of the Sth Topical Conference on RF Heating in Plasmas (University of Wisconsin, Madison 1983), p. 41. 